\title{
DESIGN AND FABRICATION OF A HTC QUASI-ONE JUNCTION SQUID BASED COMPARATOR FOR A 4-BIT SUPERCONDUCTIVE FLASH A/D CONVERTER
}

\author{
E.M.C.M. Reuvekamp, P.A.A. Booi", M.A.J. Verhoeven, G.J. Gerritsma and H. Rogalla \\ University of Twente, Department of Applied Physics, Low Temperature Division, \\ P.O. Box 217, NL-7500 AE Enschede, The Netherlands
}

\begin{abstract}
A design of a comparator for an all HTC superconductive 4-bit flash A/D converter is proposed and discussed. The design has been simulated and optimized using the PSCAN computer package $[1,2]$. The required junction and circuit parameters are thus calculated and compared with the experimental junction parameters. The operating temperature is intended to be as high as $40 \mathrm{~K}$. Due to the current noise-level at these temperatures the $I_{c}$ of the junctions should be higher than $100 \mu \mathrm{A}$. The output voltages should be of the order of $500 \mu \mathrm{V}$ and higher. The junctions in the quasi-one junction SQUID are structured on 2 ramp-edges. The operating frequencies will be below $1 \mathrm{MHz}$ in this first design. Optimization to the $\mathrm{GHz}$ range seems very well possible. A first realization uses the $\mathrm{YBa}_{2} \mathrm{Cu}_{3} \mathrm{O}_{7-x}$ (YBCO) superconductor and $\mathrm{PrBa}_{2} \mathrm{Cu}_{3} \mathrm{O}_{7-\mathrm{x}}$ (PBCO) as junction barrier and electrode inter layer material. On a $10 \times 10 \mathrm{~mm}^{2} \mathrm{SrTiO}_{3}$ (001) substrate, 9 comparators and 4 separate ramp-edge junctions are realized. This relative large number of elements on a wafer should allow us to extract statistical information on the scattering of properties due to the fabrication processes.
\end{abstract}

\section{INTRODUCTION}

The basic element in the flash A/D converter is the comparator, which is a one-junction SQUID, as proposed by Ko $[3,4]$. This device converts the analog input current to a periodic junction current $I_{J}[5]$. In order to sample the junction current, the basic one-junction SQUID is extended with an additional junction, the so-called sample junction $\mathrm{J}_{\text {. }}$ This circuit, a quasi-one junction SQUID (QOS), is drawn in fig. 1.

The basic equation for this QOS circuit, using a DC approximation, is:

$$
\varphi_{a}-\varphi_{0}=\beta_{L} \sin \varphi_{0}+\arcsin \left[\frac{I_{b}+I_{0} \sin \varphi_{0}}{I_{s}}\right]
$$

which can be approximated for low bias currents by:

$$
\varphi_{a}^{\prime}-\varphi_{0}=\beta_{L}^{\prime} \sin \varphi_{0}
$$

This research is in part supported by the European Economical Community.

*:Present address National Institute of Standards and Technology, Boulder CO 80303, USA. Manuscript received 24 August, 1992.

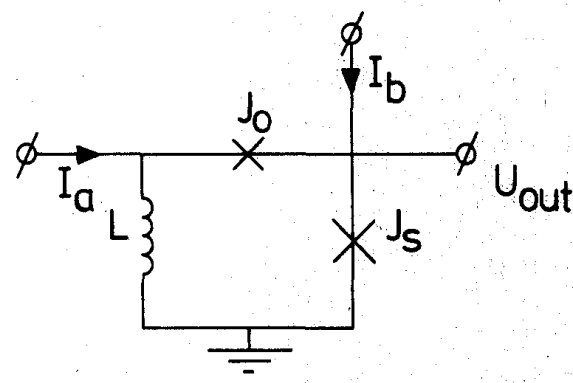

Fig. 1 Equivalent circuit of the quasi-one-junction SQUID. The junctions $J_{0}$ and $J_{s}$ have critical currents of respectively $I_{0}$ and $I_{s}$, where $I_{0}<I_{s}$.

in which

$$
\varphi_{a}^{\prime}=\varphi_{a}-\arcsin \left(\frac{I_{b}}{I_{s}}\right), \quad \beta_{L}^{\prime}=\beta_{L}+\frac{\left(\frac{I_{b}}{I_{s}}\right)}{\sqrt{\left(1-\left(\frac{I_{b}}{I_{s}}\right)^{2}\right)}}
$$

In these equations $\varphi_{0}=\sin ^{-1}\left(I_{\mathrm{y} 0} / I_{0}\right)$ is the phase of the junction $\mathrm{J}_{0}$ with critical current $\mathrm{I}_{0}, \mathrm{I}_{\mathrm{s}}$ the critical current of junction $\mathrm{J}_{\mathrm{s}}, \varphi_{\mathrm{a}}=2 \pi \mathrm{LI}_{\mathrm{a}} / \Phi_{0}$ and $\beta_{\mathrm{L}}=2 \pi \mathrm{LI}_{0} / \Phi_{0}$, where $\Phi_{0}$ is the flux quantum. The current through $J_{s}$ is sampled by periodically applying a current pulse to the bias input $\mathrm{I}_{\mathrm{b}}$. In a well trimmed circuit the pulse applied to the OOS will let $J_{\mathrm{s}}$ switch periodically with the input current to the voltage state. The current period is $\Phi_{0} \mathbf{L}$, as can be concluded from the equations above. As proposed by Ko [4], trimming can be performed by adjusting the DC-bias current such that at $I_{a}=0$ the junction $J_{s}$ switches, due to thermal activation, to a voltage state for $50 \%$ of the applied pulses.

This parallel-like A/D conversion scheme requires 4 comparators for 4-bit A/D conversion. To obtain a Graycoded output signal, the input of the $\mathrm{N}^{\mathrm{th}}$ comparator, responsible for bit $\mathrm{N}$ (where $\mathrm{N}=1$ and 4 for respectively the least and most significant bit) has to be connected to $2^{-\mathrm{N}}$ times the current to be sampled. In the next sections, an all HTC QOS design will be presented, which is similar to the designs based on the classical superconductor $\mathrm{Nb}$ $[3,4,6,7,8,9]$. The design of the input signal dividers, as well as all other elements needed in a complete $\mathrm{AD}$ converter, are beyond the work presented here. 


\section{DESIGN}

The design of a QOS operating at relatively high temperatures is facing the problem of thermally induced switching of the junction current when using a low critical current junction. The noise sensitivity is determined by the barrier energy with respect to the thermal energy $\mathrm{E}_{\mathrm{J}} / \mathrm{E}_{\mathrm{th}}=\Phi_{0} \mathrm{I}_{\mathrm{o}} / 2 \pi \mathrm{k}_{\mathrm{B}} \mathrm{T}$. For proper operation this factor should have a high value. Assuming a value of $E_{\mathrm{J}} / E_{\mathrm{th}}=100$, the critical current $I_{0}$ should be higher than 17.6, 168 and 322 $\mu \mathrm{A}$, respectively for temperatures of $4.2,40$ and $77 \mathrm{~K}$. Because the QOS threshold curve should be single valued, $\beta_{\mathrm{L}}$ should be smaller than 1, for high frequency operation in the $\mathrm{GHz}$ range preferably less than $0.5[3,4]$. From this the maximal allowed value of the loop inductance can be estimated at $1.65 \mathrm{pH}$ for $\mathrm{I}_{0}=100 \mu \mathrm{A}$. This small inductance can not be implemented by using holes or washers, because the structure containing the Josephson junctions should be of (sub)micrometer dimension. In this design an inductance implemented as a stripline above a ground plane is proposed to realise the inductance of the order of 1 picoHenry in the QOS. The thus obtained inductances can be estimated using the approach of Chang [10]. In the calculations of the stripline inductances, a penetration depth of $\lambda=145 \mathrm{~nm}$ was used for the superconductor at $40 \mathrm{~K}$. The typical inductance is estimated at $0.13 \mathrm{pH} / \mu \mathrm{m}$ for a stripline of width $w=5 \mu \mathrm{m}$, superconducting electrode thicknesses of $100 \mathrm{~nm}$ and an interspacing of also $100 \mathrm{~nm}$. The junctions are ramp type $\mathrm{YBCO} / \mathrm{PBCO} / \mathrm{YBCO}$ junctions [11] and in this QOS design located on two different ramps, as can be seen in fig. 2 . In this figure the inductance, as lumped circuit element between the two junctions, is clearly visible. For frequencies higher than $1 \mathrm{MHz}$ another design using a separate ground plane will be necessary.

According to Gao [12], the junction capacitance is estimated at $10^{-14} \mathrm{~F}$, using the RSJ model and the hysteresis of the IV curve at $4.2 \mathrm{~K}$. The capacitance parallel to the junctions, due to the two superconductive thin films separated by a thin inter layer, is much higher. This parasitic parallel capacitance is of the order $1 \mathrm{pF}$ for typical overlap areas of $10^{3}{\mu \mathrm{m}^{2}}^{2}$ and a $100 \mathrm{~nm}$ thick inter layer (using $10^{-7} \mathrm{Fcm}^{-2}$ and a dielectric constant 3 to $4 \epsilon_{0}$ ). The McCumber hysteresis parameter $\beta_{\mathrm{C}}$ has a high value due to a relative high $R_{N}$ and $C$ value. Assuming $R_{N}=10$ $100 \mathrm{Ohm}$, according to Gao [12] for this kind of junctions, $\beta_{\mathrm{C}}=30$ to $3000\left(\mathrm{I}_{0}=100 \mu \mathrm{A}, \mathrm{C}=1 \mathrm{pF}\right)$. This $\beta_{\mathrm{C}}$ value can be reduced in a future design by carefully minimizing the overlap of the electrodes.

\section{FABRICATION}

The fabrication of the quasi-one junction SQUIDs is similar to the process of single ramp type junction fabrication. An additional ex-situ process step is involved to produce the second ramp for the sample junctions $\mathrm{J}_{\mathrm{s}}$. For

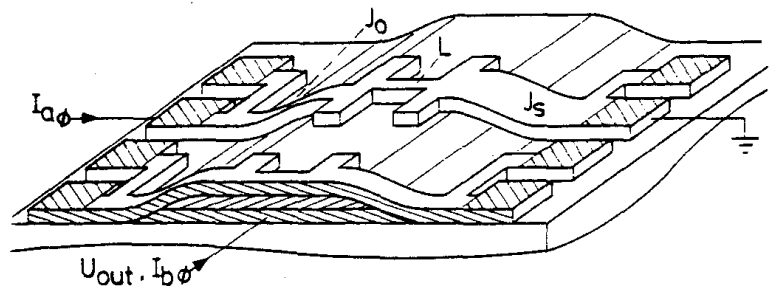

Fig. 2 Lay-out of the all HTC QOS design, based on 2 ramp type $\mathrm{YBCO} / \mathrm{PBCO} / \mathrm{YBCO}$ junctions and a stripline inductance.

Table 1. Sputter parameters

\begin{tabular}{|c|c|}
\hline Sputter pressure $(\mathrm{Pa})$ & 7 \\
\hline Gas mass flow Ar: $\mathrm{O}_{2}$ & $15: 4$ \\
\hline Heater temperature $\left({ }^{\circ} \mathrm{C}\right)$ & $\begin{array}{l}740(\mathrm{YBCO}) \\
640-740(\mathrm{PBCO})\end{array}$ \\
\hline Sputter power $(\mathrm{J} / \mathrm{s})$ & 90 \\
\hline \multicolumn{2}{|l|}{ Distances to target centre } \\
\hline $\operatorname{radial}(\mathrm{cm})$ & 8 \\
\hline $\operatorname{axial}(\mathrm{cm})$ & 4 \\
\hline Deposition rate $(\mathrm{nm} / \mathrm{hr})$ & $12-15$ \\
\hline Presputter time (hr) & $1 / 4$ \\
\hline Cool down & $\begin{array}{l}\text { in } 20 \mathrm{kPa} \mathrm{O}_{2} \text { atmosphere to } \\
400^{\circ} \mathrm{C} \text { during } 1 / 2 \mathrm{hr} \text {., to room } \\
\text { temperature during } 1 / 2 \mathrm{hr} \text {. }\end{array}$ \\
\hline
\end{tabular}

clarity, the fabrication contains the following process steps. (1) Sputter deposition of an YBCO/PBCO double layer. The settings for the YBCO/PBCO sputter deposition are presented in Tab.1. (2) Definition of the first ramp edge by photo lithography (PL) and ion-beam milling. The ionbeam milling is performed with a Kaufman source, operated in pulsed mode (on 8 sec., off $10 \mathrm{sec}$.), a beam voltage of $500 \mathrm{~V}$ and a beam current of $10 \mathrm{~mA}$. The incident angle of the beam on the sample is $45^{\circ}$, resulting in an etch rate of $5 \mathrm{~nm} /$ pulse. (3) Definition of the second ramp edge, as in step (2). (4) Ion-beam cleaning (in situ) of the ramp edges, immediately followed by sputter deposition of the PBCO junction barrier and the YBCO counter electrode. Cleaning consists of a sequence of 4 and 2 ion-beam pulses at respectively a beam voltage of 500 and 200 volt. In order to avoid contamination on the ramp edges, and thus in the junction barrier, the cleaning is performed at low angle of incidence to the substrate (about $15^{\circ}$ ) and parallel to the edges. (5) Sputter deposition of Ti/Au electrical contacts and definition by lift-off. (6) PL and ion-plasma etching through the YBCO top electrode. This last etching step is performed in a parallel plate reactor at a rf sputter power of 80 Watt (on $5 \mathrm{sec}$., off $10 \mathrm{sec}$.), a self-bias of $610 \mathrm{~V}$ and an argon pressure of $2.5 \mathrm{~Pa}$. The resulting etch rate is about $8 \mathrm{~nm} / \mathrm{min}$. In this 
final step the junctions, including voltage and current paths as well as the stripline inductance located in between the two junctions on top of the $\mathrm{PBCO}$ inter layer, are structured.

\section{RESULTS}

\section{A. Simulations}

The simulations of the quasi one-junction SQUID have been performed using the PSCAN package $[1,2]$. The units of current and voltage in the simulations were respectively $0.1 \mathrm{~mA}$ and $0.1 \mathrm{mV}$, resulting in units of resistivity, inductance, capacity, frequency and time which are respectively $1 \mathrm{Ohm}, 3.295 \mathrm{pH}, 3.295 \mathrm{pF}, 0.304 \mathrm{THz}$ and 3.25 ps. The transient response of the current through junction $\mathrm{J}_{0}$ on a sinusoidal input current $\mathrm{I}_{\mathrm{a}}$ of $100 \mathrm{MHz}$ is shown in Fig. 3. For this circuit, using the realistic parameters $\left(\beta_{\mathrm{L}}=0.5, \beta_{\mathrm{C} 0}=30, \beta_{\mathrm{Cs}}=3, \mathrm{I}_{0} / I_{\mathrm{s}}=10\right.$ and $I_{c} R_{N}=1 \mathrm{mV}$ ) as determined in the second paragraph, a smooth and nice response is visible on the sinusoidal input current with amplitude $5 \mathrm{~mA}$ (corresponding to exact $4 \Phi_{0}$ ). With the same parameters but at a frequency of $1 \mathrm{GHz}$, some oscillations of the junction current $I_{\mathrm{J} 0}$ occur after this current has changed sign and especially at moments when the input current rapidly changes value. This is also visible in Fig. 4, in which the dynamic response is presented for a sinusoidal input current $I_{a}$ of $1 \mathrm{GHz}$ and an equivalent amplitude $\Phi_{\mathrm{a}}=\mathrm{LI}_{\mathrm{a} \text { max }}=4 \Phi_{0}$. From this figure it can be observed that, although the static response curve (dashed line) shows no hysteresis, because $\beta_{\mathrm{L}}=0.5<1$, the dynamic curve at $1 \mathrm{GHz}$ (but also at a several $10 \mathrm{MHz}$ ) does. This inherent dynamic behaviour of the circuit is probably within acceptable limits up to $1 \mathrm{GHz}$, as reported by others $[4,9]$.

An example of QOS response on a sinusoidal input current $I_{a}$ and a pulsed current applied to the bias input is presented in Fig. 5. The junction parameters are the same as in Fig. 3 and 4. The frequency is $10 \mathrm{MHz}$ however, and the amplitude of the sinusoidal input current is equivalent to $1 \Phi_{0}$. The sample pulses, applied on the bias input, have a frequency of $500 \mathrm{MHz}$ (50 pulses/period, pulse width $200 \mathrm{ps}$ ) and an amplitude equal to the critical current of junction $\mathrm{J}_{\mathrm{g}}$. As can be seen in Fig. 5, the QOS responds with voltage pulses that are periodic with the input current $I_{a}$. Switching of $J_{s}$ to the voltage state, as response on an applied pulse, is observed in the dynamic simulations in particular at input currents $I_{\mathrm{a}}$ for which the flux in the QOS is about $(2 \mathrm{~m} \pm 1) \Phi_{0} / 2$. This dynamic respons on short pulses is slightly different from the expected behaviour within a static model, which also neglects the parallel current path through $\mathrm{J}_{0}$ and $\mathrm{L}$. In that case switehing of $\mathrm{J}_{s}$ to the voltage state can be expected when the current through $J_{\mathrm{s}}$ is positive, which occurs when the applied flux is $0<\Phi<\Phi_{0} / 2$ $\left( \pm \mathrm{m} \Phi_{0}\right)$. As mentioned before, fine tuning to obtain a real periodic pulsation of the QOS can be achieved by changing the pulse amplitude and the DC bias level.

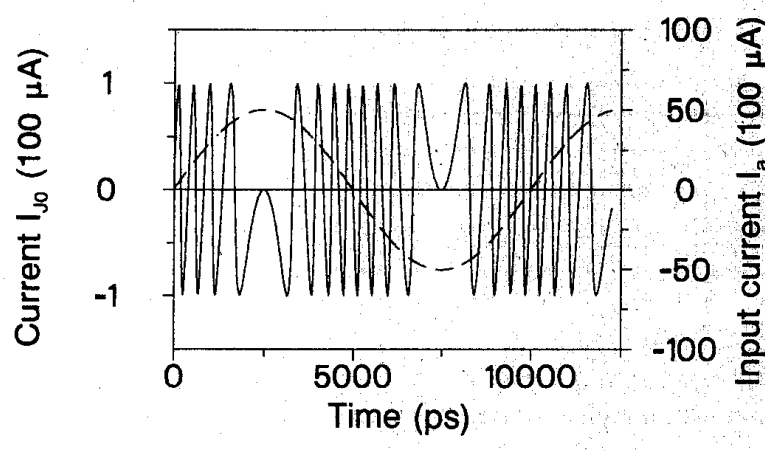

Fig. 3 Transient QOS response of the current through $\mathrm{J}_{0}$ (solid) on a $100 \mathrm{MHz}$ input current of amplitude $4 \Phi_{0} / \mathrm{L}$ (dashed). For parameters see text.

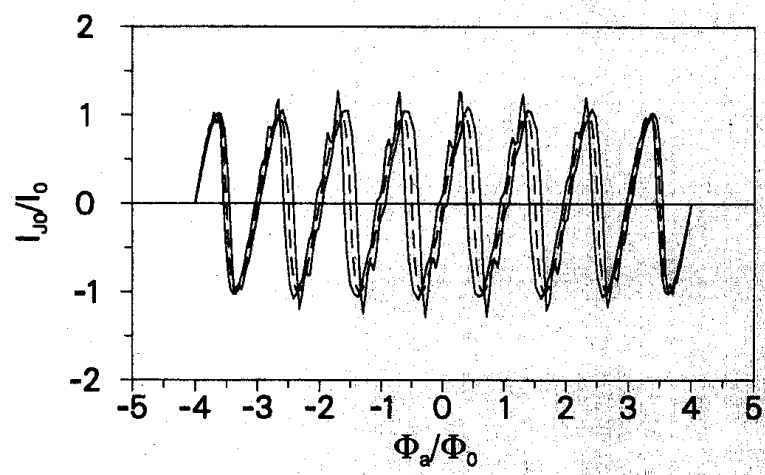

Fig. 4 Dynamic response (solid), $I_{\mathrm{j} o} / I_{0}$ versus $\Phi_{\mathrm{B}} / \Phi_{0}$ for an input current of $1 \mathrm{GHz}$ and amplitude $4 \Phi_{0} /$. The static response is dashed. For parameters see text.

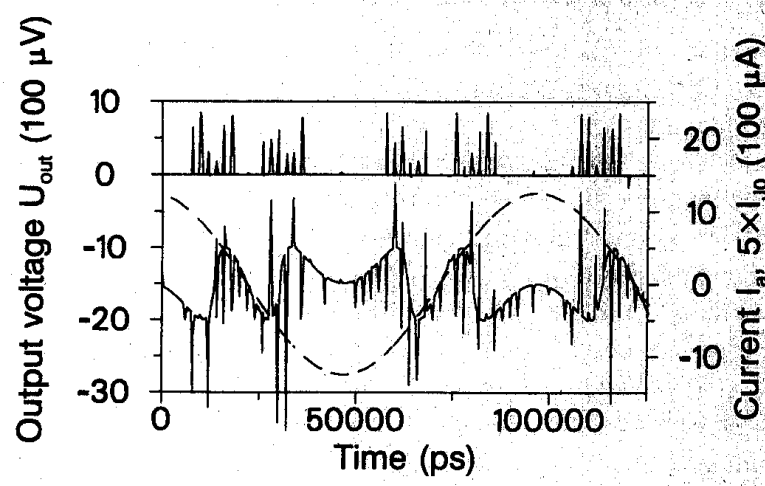

Fig. 5 QOS response on input current (dashed) of $10 \mathrm{MHz}$ and pulses on the bias input, showing output voltage pulses (upper solid). The junction current $I_{J 0}$ is given by the lower solid curve. 


\section{B. Experimental}

In a first attempt to realise a QOS it was found that it is very important to deposit particle free films of $\mathrm{YBCO}$ and PBCO. Films containing crystallites are not suited for use in a comparator of the proposed design, because of the induced shorts in the electrode overlap area and the nonreproducible and uncontrollable junction properties. As an example, the top view of a structured QOS is shown in Fig. 6. As can be seen in this figure, the $\mathrm{YBCO} / \mathrm{PBCO} / \mathrm{YBCO}$ films have many granular outgrowths. This problem can be solved by optimizing the deposition conditions as has been shown in the past $[11,12]$.

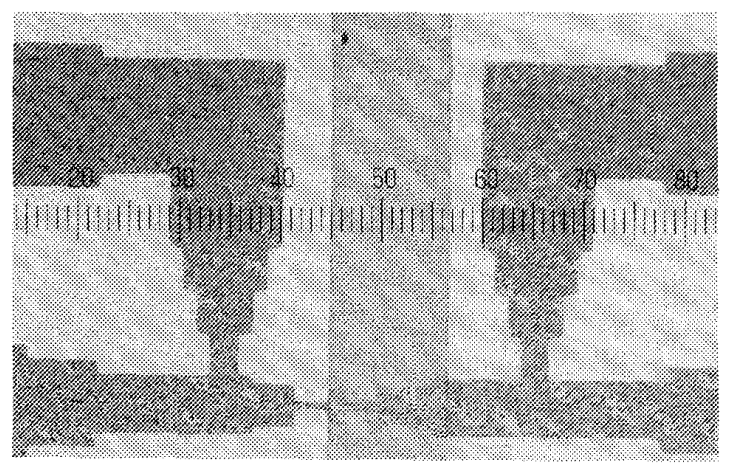

Fig. 6 Top-view on structured QOS. The junctions $J_{0}$ and $J_{s}$ are located on resp. the left and right edge. The stripline inductance is in between the junctions.

\section{SUMMARY AND CONCLUSIONS}

A design of an all HTC QOS, intended for use in an A/D converter, is presented. Ramp type junctions of $\mathrm{YBCO} / \mathrm{PBCO} / \mathrm{YBCO}$ are used. The inductance can be realised by a stripline between the junctions. Simulations by the PSCAN package show correct behaviour of the QOS up to frequencies of approximately $1 \mathrm{GHz}$. The output voltage pulses are periodic with the input current, as expected. In the dynamic simulations, output voltage pulses are observed at an applied flux of around $(2 \mathrm{~m}-1) \Phi_{0} / 2$. This value is slightly shifted compared with the value expected from a static QOS model, which predicts an applied flux value for pulse-induced switching between 0 and $\Phi_{0} / 2\left( \pm \mathrm{m} \Phi_{0}\right)$. A first realisation shows the importance of a smooth YBCO/PBCO multi layer structure. Granular films with out-growths are not applicable due to the many resulting shorts in the relative large electrode overlap area and the uncontrollable junction parameters.

\section{REFERENCES}

[1] V.K. Semenov, A.A. Odintsov and A.B. Zorin, "Automation of numerical analysis of circuits with Josephson tunnel junctions”, in SQUID'85Superconducting Quantum Interference Devices and their Applications, Berlin-New York, 1985, pp. 71-75.

[2] S.V. Polonsky, V.K. Semenov, PSCAN: Personal Superconductor Circuit ANalyzer, Moscow: Lab. of Cryoelectronics, Moscow State University, Version 1.0, October 1990, pp. 1-120.

[3] H. Ko and T. van Duzer, "A new high-speed periodicthreshold comparator for use in a Josephson A/D converter", IEEE J. Sol.-State Circuits, vol. 23, pp. 1017-1021, August 1988.

[4] H. Ko, "A flash Josephson A/D converter constructed with one-junction SQUIDS", IEEE Trans. Magn., vol. 25 , pp. 826-829, March 1989.

[5] K.K. Likharev, Dynamics of Josephson Junctions and Circuits, New York: Gordon and Breach, 1986, pp. 151-174.

[6] E.S. Fang and T.H. van Duzer, "Speed-limiting factors in flash-type josephson A/D converters", IEEE Trans. Magn., vol. 25, pp. 822-825, March 1989.

[7] P. Bradley, "Quasi-one junction SQUIDS as comparators for analog-to -digital conversion", IEEE Trans. Magn., vol. 27, pp. 2895-2897, March 1991.

[8] P. Bradley and H. Dang, "Design and testing of Quasi-one junction SQUID-based comparators at low and high speed for superconductive flash A/D convertors", IEEE Trans. Appl. Superconductivity, vol. 1, pp. 134-139, September 1991.

[9] G.T. Mailick Jr., "Computer simulations of a 20 Gigasample/second, $500 \mathrm{MHz}$ eight-bit, analog-todigital converter", IEEE Trans. Magn., vol. 27, pp. 2879-2882, March 1991.

[10] W.H. Chang, "The inductance of a superconducting strip transmission line", J. Appl. Phys., vol. 50, pp. 8129-8134, December 1979.

[11] J. Gao, W.A.M. Aarnink, G.J. Gerritsma, D. Veldhuis and H. Rogalla, "Preparation and properties of all high $T_{c}$ SNS-type edge dc SQUIDS", IEEE Trans. Magn., vol. 27, pp. 3062-3065, March 1990.

[12] J. Gao et al, "Characteristics of advanced $\mathrm{YBa}_{2} \mathrm{Cu}_{3} \mathrm{O}_{x}$ / $\mathrm{PrBa}_{2} \mathrm{Cu}_{3} \mathrm{O}_{\mathrm{x}} / \mathrm{YBa}_{2} \mathrm{Cu}_{3} \mathrm{O}_{\mathrm{x}}$ edge type junctions", Appl. Phys. Lett., vol. 59, pp. 2754-2756, November 1991. 